\title{
An Orthogonal Relay Protocol with Improved Diversity-Multiplexing Tradeoff
}

\author{
K. V. Srinivas, Raviraj Adve
}

\begin{abstract}
Cooperative relaying helps improve the reliability of data transmission over wireless fading channels. In orthogonal relay protocols the source and the relay terminals transmit over orthogonal channels, allowing a simple receiver at the destination. These protocols achieve the maximum diversity gain but suffer from reduced multiplexing gain. We propose a new orthogonal decode-andforward relay protocol that employs rotated $n$-dimensional QAM constellations for input signaling and, with a single cooperating relay, achieves a multiplexing gain of $\frac{n}{n+1}$ where $n+1$ is the cooperation frame length in symbol intervals. We show that the proposed protocol achieves a linear diversity-multiplexing tradeoff (DMT) connecting the points $\left(0, d_{\max }\right)$ and $\left(r_{\max }, 0\right)$ where $d_{\max }=$ 2 and $r_{\max }=\frac{n}{n+1}$. With $N_{R}$ relays, our proposed protocol achieves a linear DMT connecting $\left(0, N_{R}+1\right)$ and $\left(\frac{n}{n+N_{R}}, 0\right)$.
\end{abstract}

Index Terms

Cooperative relaying, Diversity, Multiplexing gain, Rotated constellations, Multi-dimensional constellations.

\section{INTRODUCTION}

In cooperative relay communications, users help each other by acting as a relay for other users signals [1], [2], [3]. Cooperative relaying creates virtual antenna arrays and realizes spatial diversity (even) with single antenna terminals. In recent times, there has been enormous interest in devising efficient cooperative relay protocols for half-duplex terminals. The cooperative protocols can broadly be classified as either orthogonal or non-orthogonal. In orthogonal protocols, the source and the relay transmit over orthogonal time intervals [3]. Due

The authors are with the Department of Electrical and Computer Engineering, University of Toronto, Toronto, Canada, M5S3G4. e-mail: \{kvsri,rsadve\}@comm.utoronto.ca. This work was supported in part by the Natural Sciences and Engineering Research Council of Canada. 
to this orthogonality, co-channel interference is avoided at the destination terminal. Though orthogonal protocols achieve the maximum diversity gain, their multiplexing gain has, so far, been limited to $1 / 2$ [4]. The tradeoff between the diversity gain and the multiplexing gain, commonly known as diversity-multiplexing tradeoff (DMT) [5], is an effective metric to evaluate the performance of relay protocols and [4] shows that the orthogonal protocols proposed in [3] achieve a linear DMT connecting maximum diversity gain of 2 with maximum multiplexing gain of $1 / 2$.

Non-orthogonal relay protocols have been proposed to improve the spectral efficiency [6], [7]. In non-orthogonal protocols, while the relay forwards the message (it received from the source) to the destination, the source transmits a new message over the same channel. This results in a multiplexing gain of 1 but the destination has to cancel co-channel interference. Recently, Pawar et al. have shown that the optimal tradeoff for the half-duplex relay channel is same as that of an equivalent multiple-input single-output (MISO) channel and proposed a non-orthogonal quantize-and-map relay protocol [8] which achieves the optimal tradeoff.

In this letter, we focus on improving the multiplexing gain (and the DMT) of orthogonal relay protocols and propose a half-duplex orthogonal decode-and-forward relay protocol that achieves a multiplexing gain higher than $1 / 2$. With a single cooperating relay, the proposed protocol has a cooperation frame of length $n+1$ symbol intervals and employs a rotated $n$-dimensional ( $n$-D) QAM constellation for input signaling. It achieves a multiplexing gain of $n /(n+1)$ and, for fixed transmission rate, it achieves the maximum diversity gain provided by the channel. When the data rate increases with SNR, we show that the diversity gain of the proposed protocol falls linearly with the multiplexing gain, resulting in a linear DMT connecting $\left(0, d_{\max }\right)$ with $\left(r_{\max }, 0\right)$, where $d_{\max }=2$ (assuming Rayleigh fading source-destination and relay-destination channels) and $r_{\max }=n /(n+1)$. The protocol can also be employed when there are $N_{R}>1$ relays assisting the transmission between a source-destination pair. In such a situation, it still achieves the maximum diversity gain $\left(\mathrm{d}_{\max }=N_{R}+1\right)$ but the multiplexing gain gets reduced to $r_{\max }=n /\left(n+N_{R}\right)$. The key contribution here is, therefore, an orthogonal protocol whose DMT is arbitrarily close to that of a non-orthogonal protocol. The cost is the use of rotated $n$-dimensional constellations.

Notation: $\Re a, \Im a, a^{*}$ and $|a|$ are real part, imaginary part, complex conjugate and absolute value of complex number $a$, respectively. $\jmath:=\sqrt{-1}$ and $E[\cdot]$ denotes expectation operator. An $n$-dimensional symbol is denoted by $\underline{x}$ and $x(i)$ denotes $i^{t h}$ component of $\underline{x}$. 


\section{System MODEL}

Consider a cooperative communication scenario in which a single relay terminal $R$ assists data transmission between source terminal $S$ and destination terminal $D$. Each terminal has a single antenna; no terminal can transmit and receive simultaneously (half-duplex constraint). Let $h_{i j}$ denote the channel gain from terminal $i$ to terminal $j, i \in\{S, R\}, j \in\{R, D\}$. We assume that $h_{S R}$ is known at $R$ and $h_{S D}, h_{S R}$ and $h_{R D}$ are known at $D .\left\{h_{i j}\right\}$ are i.i.d. with $h_{i j} \sim \mathcal{C N}(0,1)$ and we consider a block-fading environment in which the channel gains remain constant over a coherence time spanning several symbol intervals. The power constraint of the source and relay are assumed to be equal to unity and without loss of generality, we consider symbol level transmission.

The functioning of the orthogonal relay protocol of [3] with a single relay can be summarized as follows. During the first symbol interval of a cooperation frame spanning two symbol intervals, source $S$ transmits its symbol to destination $D$ and relay $R$. The relay processes the received symbol (e.g. amplify or decode and re-encode) and forwards it to $D$ in the next symbol interval while the source remains silent. This protocol is referred to as the "conventional orthogonal protocol" in the rest of the paper. The data symbol, received through two independently fading channels, $h_{S D}$ and $h_{R D}$, achieves a diversity gain of two. Only one symbol gets conveyed over two symbol intervals resulting in a maximum multiplexing gain of $1 / 2$.

\section{Proposed Orthogonal Decode-And-Forward Protocol}

In the proposed orthogonal decode-and-forward (ODF) protocol, the input symbols are chosen from a rotated $n$-D QAM constellation ${ }^{1}$. A cubic $n$-D QAM signal set $\mathcal{V}_{n \mathrm{D}}$ is obtained as the Cartesian product of $\frac{n}{2}$ 2-D QAM signal sets, i.e., $\mathcal{V}_{n \mathrm{D}}=\mathcal{V}_{2 \mathrm{D}} \times \mathcal{V}_{2 \mathrm{D}} \ldots \times \mathcal{V}_{2 \mathrm{D}}$ [9]. To achieve a data rate of $b$ bits per 2-dimensions, the $n$-D constellation will have (at least) $2^{n b / 2}$ symbols. A symbol vector from a rotated $n$-D constellation $\mathcal{X}_{n \mathrm{D}}$ is given by

$$
\underline{x}_{k}=\underline{v}_{k} \boldsymbol{\Phi}
$$

where $\underline{v}_{k}=\left(v_{k}(1), \ldots, v_{k}(n)\right) \in \mathbb{Z}^{n}$ is a symbol from un-rotated $n$-D QAM constellation $\mathcal{V}_{n \mathrm{D}}$ and $\Phi \in \mathbb{R}^{n \times n}$ is a unitary rotation matrix chosen such that that rotated symbols $\underline{x}_{k}, k=$ $1, \ldots, 2^{n b / 2}$ have the following property:

$$
x_{k}(i) \neq x_{m}(i), i=1, \ldots, n, \underline{x}_{k}, \underline{x}_{m} \in \mathcal{X}_{n \mathrm{D}}, 1 \leq k, m \leq 2^{n b / 2}, k \neq m .
$$

\footnotetext{
${ }^{1}$ The terms "constellation" and "signal set" are used synonymously.
} 
Such rotated QAM constellations were originally proposed for exploiting component (or, coordinate) level diversity available with input symbols having $n>1$ components [10], [9]. While many rotation matrices result in rotated constellations satisfying Eqn. (2), the optimal rotation matrices for up to 30-dimensions were determined and reported in [11].

A message symbol enjoys a diversity gain of two if it is received at the destination over two independent Rayleigh fading channels (in this case, the source-destination and relaydestination channels). However, this requires the relay to forward every symbol it receives from the source and, as in conventional ODF protocol, it reduces the multiplexing gain to $1 / 2$. The proposed protocol achieves a higher multiplexing gain by requiring the relay transmit only a part of the message symbol; more precisely, for each $n$-component (i.e., the $n$-D) message symbol it receives (and decodes) from the source, the relay forwards only one component to the destination. Thus, only one of the $n$ components of the message symbol experiences the two independent fading channels. If the symbols are drawn from a standard $n$-D QAM constellation, this results in a diversity gain of only one.

We chose input symbols from rotated $n$-D QAM constellations satisfying Eqn. (2) and this ensures full diversity. The DMT analysis in Section IV shows that, when the effective channel gains experienced by the components of a rotated $n$-D QAM symbol are ordered, the entire symbol enjoys a diversity gain of two even if only one of its components experiences both channels. We now detail our scheme.

Assume a cooperation frame of length $n+1$ symbol intervals and let the channel coherence time be at least as long as the cooperation frame length. With a target data rate is $b$ bits per 2-D symbol, $S$ chooses two $n$-D symbols, $\underline{x}_{k}$ and $\underline{x}_{l}$, each carrying $n b / 2$ bits of information, from a rotated $n$-D QAM signal set $\mathcal{X}_{n \mathrm{D}}$. The symbols are combined to obtain $n$ complex (equivalently, 2-D) symbols $\tilde{x}_{i}, i=1, \ldots, n$, where

$$
\tilde{x}_{i}=x_{k}(i)+\jmath x_{l}(i), i=1, \ldots, n .
$$

The transmission and reception in the proposed ODF protocol, with a single cooperating relay, are described below.

- Source $S$ transmits the symbols $\tilde{x}_{i}, i=1, \ldots, n$, in $n$ symbol intervals ${ }^{2}$. The signals received at the destination and the relay are, respectively,

$$
y_{D, i}=h_{S D} \tilde{x}_{i}+n_{D, i}, \quad i=1, \ldots, n,
$$

\footnotetext{
${ }^{2}$ The energy of the signal set $\mathcal{X}_{n \mathrm{D}}$ is scaled such that $\tilde{x}_{i}, i=1, \ldots, n$, have unit average energy.
} 


$$
y_{R, i}=h_{S R} \tilde{x}_{i}+n_{R, i}, \quad i=1, \ldots, n,
$$

where $y_{j, i}$ and $n_{j, i}$ denote the received signal and additive noise at terminal $j, j \in$ $\{D, R\}$, in the $i^{\text {th }}$ time slot and $n_{j, i} \sim \mathcal{C N}\left(0, \sigma_{j}^{2}\right)$.

- From the $n 2$-D symbols $y_{R, i}, i=1, \ldots, n, R$ obtains two $n$-D symbols $\underline{r}_{k}$ and $\underline{r}_{l}$, where

$$
\begin{aligned}
& r_{k}(i)=\Re\left(h_{S R}^{*} y_{R, i}\right)=\left|h_{S R}\right|^{2} x_{k}(i)+\Re w_{R, i}, \quad i=1, \ldots, n, \\
& r_{l}(i)=\Im\left(h_{S R}^{*} y_{R, i}\right)=\left|h_{S R}\right|^{2} x_{l}(i)+\Im w_{R, i}, \quad i=1, \ldots, n,
\end{aligned}
$$

and $w_{R, i}=h_{S R}^{*} n_{R, i}$.

- $R$ decodes ${ }^{3}$ the symbols $\underline{r}_{k}$ and $\underline{r}_{l}$ :

$$
\underline{\hat{x}}_{j}=\left.\left.\arg \min _{\underline{x}_{q} \in \mathcal{X}_{n \mathrm{D}}}\left|\underline{r}_{j}-\right| h_{S R}\right|^{2} \underline{x}_{q}\right|^{2}, j \in\{k, l\} .
$$

- From $\underline{\hat{x}}_{k}$ and $\underline{\hat{x}}_{l}, R$ forms $\hat{\tilde{x}}_{1}=\hat{x}_{k}(1)+\jmath \hat{x}_{l}(1)$ and forwards to the destination in the $(n+1)^{t h}$ time slot. Assuming that $R$ decodes the symbols correctly (i.e., $\underline{\hat{x}}_{k}=\underline{x}_{k}$ and $\left.\underline{\hat{x}}_{l}=\underline{x}_{l}\right), D$ receives,

$$
y_{D, n+1}=h_{R D} \tilde{x}_{n+1}+n_{D, n+1} .
$$

- Using Eqns. (4) and (6), $D$ generates two $n$-D symbols $\underline{y}_{k}$ and $\underline{y}_{l}$, where

$$
\begin{gathered}
y_{k}(1)=\Re\left(h_{S D}^{*} y_{D, 1}\right)+\Re\left(h_{R D}^{*} y_{D, n+1}\right)=\gamma x_{k}(1)+\Re w_{D, 1}+\Re w_{D, n+1}, \\
y_{l}(1)=\Im\left(h_{S D}^{*} y_{D, 1}\right)+\Im\left(h_{R D}^{*} y_{D, n+1}\right)=\gamma x_{l}(1)+\Im w_{D, 1}+\Im w_{D, n+1},
\end{gathered}
$$

and

$$
\begin{gathered}
y_{k}(i)=\Re\left(h_{S D}^{*} y_{D, i}\right)=\left|h_{S D}\right|^{2} x_{k}(i)+\Re w_{D, i}, \quad i=2, \ldots, n, \\
y_{l}(i)=\Im\left(h_{S D}^{*} y_{D, i}\right)=\left|h_{S D}\right|^{2} x_{l}(i)+\Im w_{D, i}, \quad i=2, \ldots, n .
\end{gathered}
$$

where $\gamma=\left|h_{S D}\right|^{2}+\left|h_{R D}\right|^{2}$ and

$$
\begin{aligned}
& w_{D, i}=h_{S D}^{*} n_{D, i}, i=1, \ldots, n, \\
& w_{D, n+1}=h_{R D}^{*} n_{D, n+1} .
\end{aligned}
$$

- From $\underline{y}_{k}$ and $\underline{y}_{l}, D$ obtains $\underline{\hat{x}}_{k}$ and $\underline{\hat{x}}_{l}$ :

$$
\begin{aligned}
\underline{\hat{x}}_{j}=\arg \min _{\underline{x}_{q} \in \mathcal{X}_{n \mathrm{D}}} \mid\left(y_{j}(1), y_{j}(2), \ldots, y_{j}(n)\right)- & \\
& \left.\left(\gamma x_{q}(1),\left|h_{S D}\right|^{2} x_{q}(2), \ldots,\left|h_{S D}\right|^{2} x_{q}(n)\right)\right|^{2}, j \in\{k, l\} .
\end{aligned}
$$

${ }^{3}$ As we are considering uncoded transmission, it is equivalent to demodulating the symbol. 
As can be observed, $S$ transmits during the first $n$ symbol intervals and $R$ transmits only during the $(n+1)^{t h}$ symbol interval. As it takes $n+1$ symbol intervals for $n$ symbols, the protocol achieves a maximum multiplexing gain of $n /(n+1)$. When there are $N_{R}>1$ relays, relay $R_{i}, i=1, \ldots, N_{R}$, forwards symbol $\hat{\tilde{x}}_{1}$ to $D$ in $(n+i)^{\text {th }}$ symbol interval and the maximum multiplexing gain gets reduced to $n /\left(n+N_{R}\right)$. In the next section, for the case of $N_{R}=1$, we determine the diversity gain when the multiplexing gain increases from 0 to $n /(n+1)$.

\section{Diversity-MultipleXing Gain TradeofF AnAlysis}

The DMT is essentially the tradeoff, at high signal-to-noise ratio (SNR), between the error probability and the data rate of a system [5]. A diversity gain $d(r)$ is said to be achieved at a multiplexing gain of $r$, if the transmission data rate $\mathrm{R}$ scales as $\mathrm{R}(\mathrm{SNR})=r \log \mathrm{SNR} b / \mathrm{s} / \mathrm{Hz}$ and the error probability scales as, $P_{s} \approx \mathrm{SNR}^{-\mathrm{d}(\mathrm{r})}$. More precisely, the multiplexing gain $\mathrm{r}$ and the corresponding diversity gain $d(r)$ are defined as

$$
\begin{gathered}
\lim _{\mathrm{SNR} \rightarrow \infty} \frac{\mathrm{R}(\mathrm{SNR})}{\log \mathrm{SNR}}=\mathrm{r}, \\
\lim _{\mathrm{SNR} \rightarrow \infty} \frac{\log P_{s}(\mathrm{SNR})}{\log \mathrm{SNR}}=-\mathrm{d}(\mathrm{r}) .
\end{gathered}
$$

Assume that two arbitrary symbols $\underline{x}_{k}, \underline{x}_{l} \in \mathcal{X}_{n \mathrm{D}}$ are transmitted as described in Section III. With uncoded data transmission, we compute the probability that one of the two $n$-D symbols transmitted in a cooperation frame gets decoded erroneously when the cardinality of the QAM signal set increases with SNR to support a data rate of $\mathrm{R}=r \log \mathrm{SNR} b / \mathrm{s} / \mathrm{Hz}$, $0 \leq \mathrm{r} \leq \frac{n}{n+1}$. We denote this probability by $P_{s, 1}$.

Let $\operatorname{Pr}\left\{\underline{x}_{k} \rightarrow \underline{x}_{m}\right\}$ be the pairwise error probability (PEP) of confusing $\underline{x}_{k}$ with $\underline{x}_{m}$ when $\underline{x}_{k}$ and $\underline{x}_{m}$ are the only two hypotheses. At high SNRs,

$$
\operatorname{Pr}\left\{\underline{x}_{k} \rightarrow \underline{x}_{\eta_{k}}\right\}<\operatorname{Pr}\left\{\operatorname{error} \mid \underline{x}_{k} \text { sent }\right\} \leq \sum_{\underline{x}_{m} \in \mathrm{X}_{k}} \operatorname{Pr}\left\{\underline{x}_{k} \rightarrow \underline{x}_{m}\right\}
$$

where $\underline{x}_{\eta_{k}}$ denotes a nearest neighbor to $\underline{x}_{k}$ and $\mathrm{X}_{k} \subset \mathcal{X}_{n \mathrm{D}}$ is the set of all nearest neighbors of $\underline{x}_{k}$.

$$
\operatorname{Pr}\left\{x_{k} \rightarrow x_{m} \mid h_{S D}, h_{R D}\right\}=Q\left(\frac{\left|u_{k}-u_{m}\right|}{2 \sigma}\right)
$$

where $Q(\cdot)$ is the Gaussian $Q$-function [12] and

$$
\underline{u}_{j}=\left(\gamma x_{j}(1),\left|h_{S D}\right|^{2} x_{j}(2), \ldots,\left|h_{S D}\right|^{2} x_{j}(n)\right), j \in\{k, m\} .
$$




$$
\Rightarrow\left|\underline{u}_{k}-\underline{u}_{m}\right|=\sqrt{\gamma^{2} \alpha_{k m}(1)+\left(\left|h_{S D}\right|^{2}\right)^{2} \sum_{i=2}^{n} \alpha_{k m}(i)},
$$

where $\alpha_{k m}(i)=\left(x_{k}(i)-x_{m}(i)\right)^{2}$. As the constellation is rotated such that no two symbols have a common coordinate (cf. Eqn. (2)), $\alpha_{k m}(i)>0, i=1, \ldots, n$, and using the fact that $\gamma^{2}=\left|h_{S D}\right|^{2}+\left|h_{R D}\right|^{2}>\left|h_{S D}\right|^{2}$

$$
\sqrt{\gamma^{2} \alpha_{k m}(1)}<\left|\underline{u}_{k}-\underline{u}_{m}\right|<\sqrt{\gamma^{2} \sum_{i=1}^{n} \alpha_{k m}(i)} .
$$

From Eqn. (1), $x_{k}(i)=\sum_{t=1}^{n} \phi_{i t} v_{k}(t)$ and $x_{m}(i)=\sum_{t=1}^{n} \phi_{i t} v_{m}(t)$ and hence,

$$
\alpha_{k m}(i)=\sum_{t=1}^{n} \phi_{i t}^{2}\left(v_{k}(t)-v_{m}(t)\right)^{2} .
$$

As the proposed protocol requires $n+1$ symbol intervals for $n 2$-D symbols, to meet a transmission data rate of $\mathrm{R}=\mathrm{r} \log \mathrm{SNR} \mathrm{b} / \mathrm{s} / \mathrm{Hz}$ (per 2-D), each of the 2-D symbols should have $(n+1) \mathrm{R} / n$ bits of information. This requires that the $n$-D symbols $\underline{x}_{k}$ and $\underline{x}_{l}$ carry $(n+1) \mathrm{R} / 2$ bits each, and hence, the $n$-D constellation will have $2^{(n+1) \mathrm{R} / 2 n}$ points in each of the $n$ dimensions. The distance between two adjacent points is $2^{-(n+1) \mathrm{R} / 2 n}=\mathrm{SNR}^{-(n+1) r / 2 n}[5]$. If $\underline{v}_{k}$ and $\underline{v}_{m}$ are $d_{k m}^{t}$ symbols apart along $t^{t h}$ dimension, $1 \leq t \leq n$,

$$
\left(v_{k}(t)-v_{m}(t)\right)^{2}=\left(d_{k m}^{t}\right)^{2} \mathrm{SNR}^{-(n+1) r / n} .
$$

Note that $d_{k m}^{t}$ is non-zero for at least one value of $t$ when $k \neq m$. This implies,

$$
\alpha_{k m}(i)=\sum_{t=1}^{n} \phi_{i t}^{2}\left(d_{k m}^{t}\right)^{2} \operatorname{SNR}^{-(n+1) r / n} .
$$

The noise term in $\underline{y}_{k}$ is $\left(\Re\left(h_{S D}^{*} n_{D, 1}\right)+\Re\left(h_{R D}^{*} n_{D, n+1}\right), \Re\left(h_{S D}^{*} n_{D, 2}\right), \ldots, \Re\left(h_{S D}^{*} n_{D, n}\right)\right)$ (cf. Eqn. (9)). For a given value of $h_{S D}$ and $h_{R D}$, its variance $\sigma^{2}$ can be bounded as

$$
\gamma \frac{\sigma_{D}^{2}}{2}<\sigma^{2}<n \gamma \frac{\sigma_{D}^{2}}{2}
$$

Using Eqns. (15), (16) and (17) in Eqn. (12), and by noting that $1 / \sigma_{D}^{2}=\mathrm{SNR}$, we bound the PEP as

$$
E_{\gamma}\left[Q\left(\sqrt{\gamma \operatorname{SNR}^{(1-(n+1) r / n)} C_{1}}\right)\right]<\operatorname{Pr}\left\{\underline{x}_{k} \rightarrow \underline{x}_{m}\right\}<E_{\gamma}\left[Q\left(\sqrt{\gamma \operatorname{SNR}^{(1-(n+1) r / n)} C_{2}}\right)\right],
$$

where $C_{1}=\left(\sum_{i=1}^{n} \sum_{t=1}^{n} \phi_{i t}^{2}\left(d_{k m}^{t}\right)^{2}\right) / 2$ and $C_{2}=\left(\sum_{t=1}^{n} \phi_{i t}^{2}\left(d_{k m}^{t}\right)^{2}\right) / 2 n$.

Now, since $\gamma=\left|h_{S D}\right|^{2}+\left|h_{R D}\right|^{2}$ is a chi-square random variable with four degrees of freedom, its distribution is given by $f(\gamma)=\frac{1}{\Gamma(2)} \gamma e^{-\gamma}$ [12]. Using $f(\gamma)$, the upper and lower bounds in Eqn. (18) can be evaluated to show that,

$$
C_{k m}^{L} \mathrm{SNR}^{-2(1-(n+1) \mathrm{r} / n)}<\operatorname{Pr}\left\{\underline{x}_{k} \rightarrow \underline{x}_{m}\right\}<C_{k m}^{U} \mathrm{SNR}^{-2(1-(n+1) \mathrm{r} / n)}
$$


where $C_{k m}^{L}$ and $C_{k m}^{U}$ are appropriately defined constants. As both the upper bound and the lower bound on $\operatorname{Pr}\left\{\underline{x}_{k} \rightarrow \underline{x}_{m}\right\}$ have the same SNR exponent

$$
\operatorname{Pr}\left\{\underline{x}_{k} \rightarrow \underline{x}_{m}\right\}=C_{k m} \mathrm{SNR}^{-2(1-(n+1) \mathrm{r} / n)},
$$

where $C_{k m}$ represents the coding gain. By substituting the PEP obtained above in Eqn. (11), we get $\operatorname{Pr}\left\{\operatorname{error} \mid \underline{x}_{k}\right.$ sent $\}=C_{k} \mathrm{SNR}^{-2(1-(n+1) \mathrm{r} / n)}$, and hence

$$
P_{s, 1}=\sum_{\underline{x}_{k} \in \mathcal{X}_{n \mathrm{D}}} \operatorname{Pr}\left\{\text { error } \mid \underline{x}_{k} \text { sent }\right\}=\operatorname{CSNR}^{-2(1-(n+1) \mathrm{r} / n)} .
$$

$P_{s, 2}$, the error probability of the second $n$-D symbol transmitted in the cooperation frame, can be computed in a similar manner and it can be shown that $P_{s, 2}=P_{s, 1}$. Hence, the symbol error probability in the proposed protocol is given by $P_{s}=\operatorname{CSNR}^{-2(1-(n+1) r / n)}$. Thus, conditioned on relay correctly decoding the symbols received from the source, the DMT of the proposed protocol is given by the following result.

In the proposed orthogonal demodulate-and-forward protocol, the diversity gain scales with the multiplexing gain as

$$
\mathrm{d}(\mathrm{r})=2(1-(n+1) \mathrm{r} / n), \mathrm{r} \in[0, n /(n+1)] .
$$

With $N_{R}>1$ relays, the DMT can be obtained in a similar manner. In this case, $\gamma=$ $\left|h_{S D}\right|^{2}+\sum_{i=1}^{N_{R}}\left|h_{R_{i} D}\right|^{2}$ and the resulting DMT is given by

$$
\mathrm{d}(\mathrm{r})=\left(N_{R}+1\right)\left(1-\left(n+N_{R}\right) \mathrm{r} / n\right), \mathrm{r} \in\left[0, n /\left(n+N_{R}\right)\right] .
$$

It is worth noting that, instead of $\hat{\tilde{x}}_{i}$, the relay can as well transmit $\hat{\tilde{x}}_{i}=\hat{x}_{k}(i)+\jmath \hat{x}_{l}(i)$ where $i$ takes any value between 1 to $n$. In such a case, the corresponding $\alpha_{k m}(i)$ would get multiplied by $\gamma$ giving exactly the same results as above.

It is known that a diversity gain of $n$ can be obtained by transmitting the $n$ components of a QAM symbol satisfying Eqn. (2) over $n$ independent Rayleigh fading channels [10], [9]. However, in the proposed protocol, $\left|h_{S D}\right|^{2}+\left|h_{R D}\right|^{2}$ and $\left|h_{S D}\right|^{2}$, the channel gains experienced by the first and the remaining components, respectively, of the symbol are not independent. Our analytical results shows that, in such a case, the diversity gain of the symbol is determined by the maximum among the diversity gains of its components. This is because in atated constellation, each coordinate is unique. 


\section{Discussion AND CONCLUSIONS}

Pawar et al. [8] have shown that the optimal DMT of a half-duplex cooperating relay network with $N_{R}$ relays is given by

$$
\mathrm{d}^{*}(\mathbf{r})=\left(N_{r}+1\right)(1-\mathrm{r}), \mathbf{r} \in[0,1]
$$

which is same as the DMT of an $\left(N_{R}+1\right) \times 1$ MISO system. These authors also proposed a non-orthogonal quantize-and-map protocol that achieves the optimal DMT. Earlier, several relay schemes have been proposed to improve the multiplexing gain and the DMT of halfduplex relaying [13], [14], [15], [16]. While [14], [15] proposed non-orthogonal protocols, [13] assumes no direct channel between the source and the destination. Tannious et al. [16] proposed an orthogonal decode-and-forward protocol that achieves the MISO DMT. However, the work in [16] assumes feedback from the destination.

For $N_{R}=1$, Fig. 1 compares the DMT of the proposed protocol with that of a conventional ODF protocol and the optimal DMT (which is nothing but the DMT of a $2 \times 1$ MISO system). For $n=1$, the proposed protocol is equivalent to that of a conventional ODF protocol and with increasing $n$, the DMT of the proposed protocol gets closer to the $2 \times 1$ MISO DMT. Thus, the proposed orthogonal protocol outperforms the existing orthogonal protocols and achieves a DMT that is very close to the optimal DMT which is shown to be achievable by a non-orthogonal protocol.

For $N_{R}>1$, the DMT of a conventional orthogonal protocol is given by [4]

$$
\mathrm{d}(\mathrm{r})=\left(N_{R}+1\right)\left(1-\left(N_{R}+1\right) \mathrm{r}\right), \mathrm{r} \in\left[0,1 /\left(N_{R}+1\right)\right]
$$

Comparing it with Eqn. (22), we can see that the proposed protocol outperforms the conventional one for every $n>1$.

It is to be noted that the improved DMT performance comes at a price of decoding complexity. In each cooperation frame of length $n$, the relay and the destination have to decode two $n$-D QAM symbols rather than $n$ 2-D QAM symbols as in a conventional ODF protocol. Furthermore, the use of a rotated constellation allows for full diversity order with only one symbol relayed. The high SNR analysis here does not account for the resulting SNR penalty to achieve a target error rate at some finite SNR. 


\section{REFERENCES}

[1] A. Sendonaris, E. Erkip, and B. Aazhang, "User cooperation diversity part I: System description," IEEE Trans. Commun., vol. 51, pp. 1927-1938, Nov 2003.

[2] A. Sendonaris, E. Erkip, and B. Aazhang, "User cooperation diversity part II: Implementation aspects and performance analysis,” IEEE Trans. Commun., vol. 51, pp. 1939-1948, Nov 2003.

[3] J. N. Laneman, D. N. C. Tse, and G. W. Wornell, "Cooperative diversity in wireless networks: Effi cient protocols and outage behavior," IEEE Trans. Inf. Theory, vol. 50, pp. 3062-3080, Dec 2004.

[4] J. N. Laneman and G. W. Wornell, "Distributed space-time coded protocols for exploiting cooperative diversity in wireless networks," IEEE Trans. Inf. Theory, vol. 49, pp. 2415-2525, Oct 2003.

[5] L. Zheng and D. N. C. Tse, "Diversity and multiplexing: A fundamental tradeoff in multiple antenna channels," IEEE Trans. Inf. Theory, vol. 49, pp. 1073-1096, May 2003.

[6] R. Nabar, H. Bolcskei, and F. W. Kneubhler, "Fading relay channels: Performance limits and space-time signal design," IEEE J. Sel. Areas Commun., vol. 22, pp. 1099-1109, Aug. 2004.

[7] K. Azarian, H. E. Gamal, and P. Schniter, "On the achievable diversity multiplexing tradeoff in half-duplex cooperative channels," IEEE Trans. Inf. Theory, vol. 51, pp. 4152-4172, Dec 2005.

[8] S. Pawar, A. S. Avestimehr, and D. N. C. Tse, "Diversity-multiplexing tradeoff of the half-duplex relay channel," in Proc. $46^{\text {th }}$ Annual Allerton Conf., Monticello, IL, Sept. 2008, p. Available at http://arxiv.org/abs/0810.2133.

[9] J. Boutrous and E. Viterbo, "Signal space diversity: A power and bandwidth efcient diversity technique for rayleigh fading channel," IEEE Trans. Inf. Theory, vol. 44, pp. 1453-1467, Jul. 1998.

[10] K. Boulle and J. C. Belfi ore, "Modulation schemes designed for the rayleigh channel," in Proc. Conference on Information Sciences and Systems (CISS), Princeton, NJ, Mar. 1992, pp. 288-293.

[11] E. Viterbo, Table of best known full diversity algebraic rotations, Available at http://www1.tlc.polito.it/ viterbo/rotations/rotations.html.

[12] M. K. Simon and M.-S. Alouini, Digital Communication over Fading Channels, John Wiley \& Sons Inc., 2005.

[13] B. Rankov and A. Wittneben, "Spectral effi cient signaling for halh-duplex relay channels," in Proc. Asilomar Conf. Signals, Syst., Comput., Pacifi c Grove, CA., Nov. 2005, pp. 1066-1071.

[14] S. Yang and J.-C. Belfi ore, “Towards the optimal amplify-and-forward cooperative diversity scheme," IEEE J. Sel. Areas Commun., vol. 53, pp. 3114-3126, Sept. 2007.

[15] Y. Fan, C. Wang, J. Thompson, and H.V. Poor, "Recovering multiplexing loss through successive relaying using repetition coding," IEEE Trans. Wireless Commun., vol. 6, pp. 4484-4493, Dec. 2007.

[16] R. Tannious and A. Nosratinia, "Spectrally effi cient relay selection with limited feedback," IEEE J. Sel. Areas Commun., vol. 26, pp. 1419-1428, Oct. 2008. 


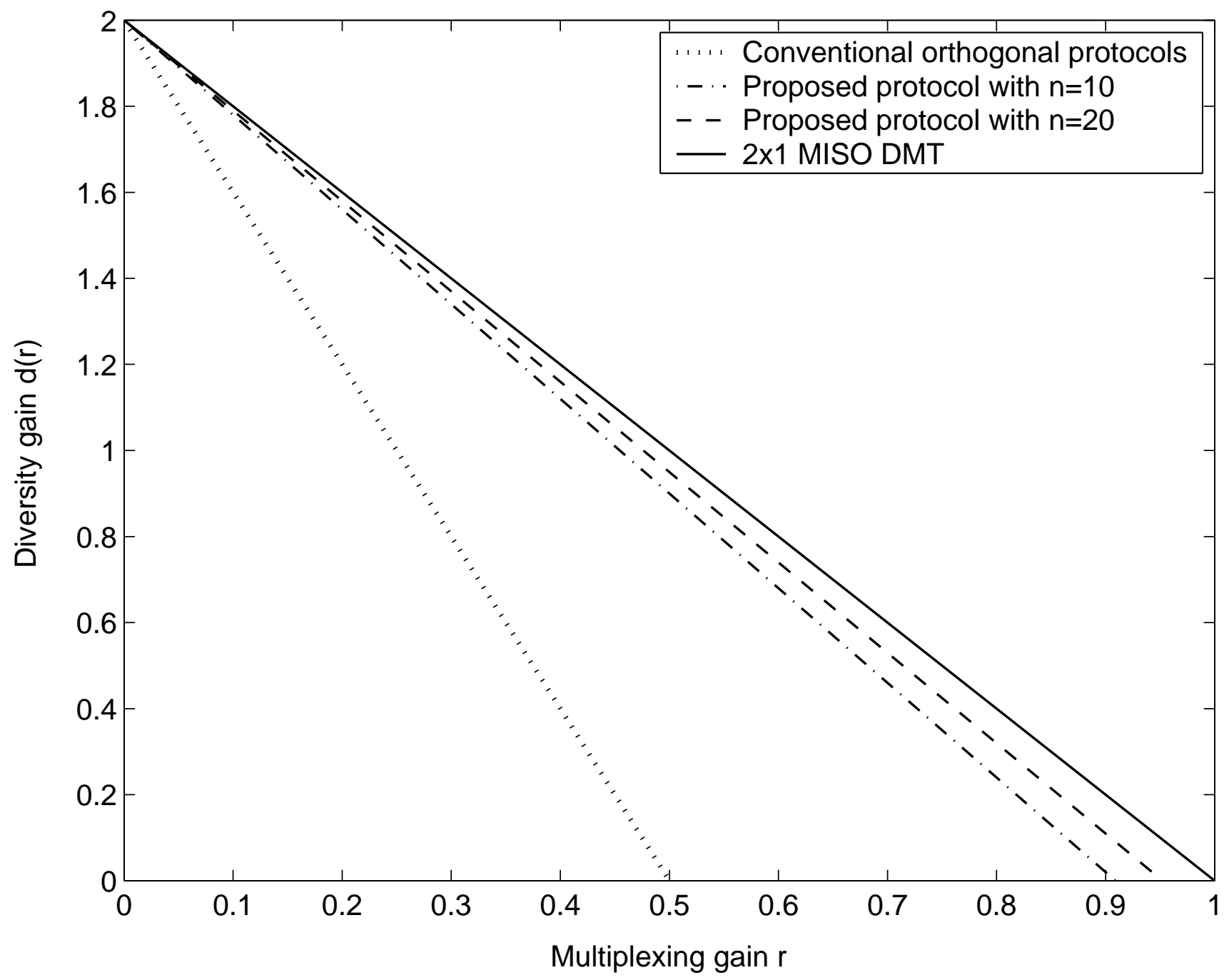

Fig. 1. Diversity-multiplexing tradeoff with a single relay. 